\title{
A note on the measurement of stimulus discriminability in conditional discriminations
}

\author{
K. GEOFFREY WHITE, MARGARET-ELLEN PIPE, and ANTHONY P. McLEAN \\ Victoria University of Wellington, Wellington, New Zealand
}

\begin{abstract}
In a conditional discrimination, pigeons' responses on a right key were reinforced in the presence of a $0^{\circ}$ line orientation, and responses on a left key were reinforced in the presence of any of five other orientations, $15^{\circ}$ through $75^{\circ}$. Variable-interval schedules of reinforcement for right and left responses were changed over five experimental conditions. Values for a measure of discriminability of $0^{\circ}$ from other orientations increased as orientation difference increased. Sensitivity of the choice between right and left in the different orientations, to reinforcers for right and left responses, changed with orientation difference. The effect of reinforcers in one component of a conditional discrimination on responding in another may therefore be modulated by the discriminability of the stimuli signaling the components.
\end{abstract}

In a simple successive discrimination, two stimuli alternate in succession, each associated with a reinforcement schedule. A discrimination may develop as long as the stimuli and their associated schedules differ. The most obvious evidence that the discrimination depends on differential reinforcement is the absence of differential responding when the reinforcer rates are the same in the presence of the two stimuli. We have argued that if the reinforcer differential is varied over several experimental conditions, it is possible to obtain an index of the extent of control by the discriminative stimuli independently of the effects of the reinforcer differentials that otherwise maintain the discrimination (White, Pipe, \& McLean, 1983, 1984). This index is the exponent of the power function relating ratios of responses in the two stimuli to ratios of reinforcers.

Traditional methods for obtaining measures of discriminability of successively presented stimuli independently of reinforcement effects rely on successive conditional discriminations. For example, standard detection procedures involve discrete trials in which the stimuli to be discriminated are presented successively across trials and the choice or detection response is made within trials. Because the choice response involves a simultaneous discrimination (e.g., between left and right, or red and green, or yes and no), the detection procedure introduces an additional discrimination. That is, detection and other conditional procedures require discriminations between successive stimuli and also between the simultaneous stimuli signaling the choice response (White et al., 1984). The advantage of the additional simultaneous discrimination is that it allows errors to occur. In the context of traditional treatments of detection performance, the occurrence of errors is essential if analyses are designed to separate

The authors' mailing address is: Department of Psychology, Victoria University of Wellington, Wellington, New Zealand. the effects of stimulus discriminability from response bias toward reporting one or another stimulus.

In our earlier work (White et al., 1983), we had found that, as stimulus separation between successively presented line orientations increased, the exponent of the power function relating ratios of responses in the different line orientations to ratios of reinforcers also increased. The purpose of the present study was to repeat the first experiment of the earlier study, using a conditional discrimination that allowed a more traditional approach to the problem of measuring stimulus discriminability. In the earlier study, $0^{\circ}$ line was alternated with other line orientations of $15^{\circ}$ through $75^{\circ}$ in $15^{\circ}$ steps. One variableinterval (VI) schedule was arranged in $0^{\circ}$, and another was associated with the other orientations. All line orientations were presented on a single key. In the present experiment, identical line orientations were presented simultaneously on both left and right keys in a two-key chamber. The reinforcement schedules were the same as in the earlier study, but responses on the right key were reinforced according to one VI schedule if the orientation was $0^{\circ}$, and left-key responses were reinforced according to another VI schedule if any other line orientation was displayed. Thus, our procedure was a standard free-operant conditional discrimination in which successively presented stimuli varied within sessions and reinforcement schedules varied between conditions. We asked whether stimulus discriminability measured according to the more traditional approach varied in the same way as it did in the simple successive discrimination in the study by White et al. (1983).

\section{METHOD}

\footnotetext{
Subjects

Two adult homing pigeons (Birds G4 and G5) with prior experimental histories of line-tilt discrimination were maintained at $80 \pm 5 \%$ of their free-feeding weights. Grit and water were available in the living cages, and supplementary feeding ensured maintenance of the prescribed weights.
} 


\begin{abstract}
Apparatus
A Coulbourn Instruments experimental chamber was enclosed in a light- and sound-attenuating cubicle. A ventilation fan provided effective masking noise. Two translucent response keys, $2.4 \mathrm{~cm}$ in diameter, were mounted on the left and right, $16 \mathrm{~cm}$ apart and $20 \mathrm{~cm}$ above the grid floor. Access to wheat for $3 \mathrm{sec}$ was possible via a central hopper opening. Each key could be operated by a force of $0.2 \mathrm{~N}$, and each response on either key produced a feedback relay click. Single $2-\mathrm{mm}$ wide white-on-black lines, $1.8 \mathrm{~cm}$ long and oriented $0^{\circ}, 15^{\circ}, 30^{\circ}, 45^{\circ}$, $60^{\circ}$, or $75^{\circ}$ from vertical, could be presented on the keys by means of in-line displays mounted directly behind the keys. At any one time, lines with the same orientation were displayed on both keys. The brightness of the lines was about $2 \mathrm{~cd} / \mathrm{m}^{2}$ and did not vary with orientation. A houselight located at the top center of the intelligence panel and reflected toward the ceiling dimly illuminated the chamber during each session. Experimental events were controlled and recorded by solidstate equipment located in an adjacent room.
\end{abstract}

\section{Procedure}

Following 4 days of preliminary training in which pecking on both keys at the specific stimuli used in the present experiment was established, the birds were introduced to the main procedure. Each session comprised 6050 -sec components. Presentations of the $0^{\circ}$ line orientation simultaneously on left and right keys alternated directly with orientations of $15^{\circ}$ through $75^{\circ}$ (in $15^{\circ}$ steps) presented on both keys. Orientations of $15^{\circ}$ through $75^{\circ}$ were presented in a random order that recycled in each block of 30 components. The $0^{\circ}$ orientation occurred five times more frequently than each other orientation, because it was directly alternated with the other orientations. There was no blackout between components, and the key was dark during reinforcements.

In the presence of the $0^{\circ}$ orientation on both keys, right-key responses were reinforced according to one VI schedule and left-key responses were never reinforced (extinction). In the presence of any of the other orientations (displayed on both keys), left-key responses were reinforced according to a different VI schedule and right-key responses were never reinforced. The VI schedules were constructed from constant-probability progressions, and reinforcements set up but not obtained at the end of a component were available when that component was next presented.

In the first condition, the schedules in both components were VI $60 \mathrm{sec}$ (VI 60) and the condition lasted for 60 sessions for each bird. Subsequent conditions were conducted for 20 sessions each. The values of the VI schedules (in seconds) for the subsequent conditions (in order of conduct) for the successively presented components were: VI 60 VI 120; VI 60 VI 240; VI 120 VI 60; VI 240 VI 60.

\section{RESULTS AND DISCUSSION}

Responses on the left and right in the presence of each line orientation were summed over the last 5 days of each reinforcement condition. The data were treated according to the analysis of signal detection procedures proposed by Davison and Tustin (1978). The generalized matching law for choice in concurrent schedules (Baum, 1974) predicts that ratios of left and right responses change as a power function of the ratio of reinforcers obtained by the responses. In the present case, the subject could choose to respond on left or right keys during the 60 -sec presentation of each line orientation (displayed on both keys). According to Davison and Tustin's (1978) first main assumption, the tendency toward responding left or right is determined by the reinforcers for left versus right responses over all line orientations. Their second main assumption is that responding is biased by stimulus discriminability. That is, if the $0^{\circ}$ orientation can be discriminated from the others, there will be a bias (d) toward right responses that is constant over all reinforcement condi- tions. The prediction for the ratio of right to left responses $\left(\mathrm{P}_{\mathrm{R}} / \mathrm{P}_{\mathrm{L}}\right)$ in the presence of the $0^{\circ}$ orientation is therefore (in logarithmic terms):

$$
\log \left(\frac{P_{R}}{P_{L}}\right)=a_{r 1} \log \left(\frac{R_{R}}{R_{L}}\right)+\log c+\log d_{1} .
$$

In Equation $1, R_{R}$ and $R_{L}$ are total reinforcers obtained by right and left responses, respectively, for all line orientations, and $a_{r}$, the exponent of the power function, describes the senstivity of response ratios to changes in reinforcer ratios. Log c is a parameter ("inherent bias") that remains constant over all experimental conditions and describes an overall constant tendency toward choosing right versus left.

The predictions for the right-to-left response ratios in the presence of each of the other orientations are made in exactly the same way, except that the constant that describes stimulus discriminability, $\log \mathrm{d}_{2}$, has the opposite sign, because in orientations other than $0^{\circ}$ there is a tendency toward choosing left, depending on how well the other orientations can be discriminated from $0^{\circ}$. That is,

$$
\log \left(\frac{P_{R}}{P_{L}}\right)=a_{r 2} \log \left(\frac{R_{R}}{R_{L}}\right)+\log c-\log d_{2} .
$$

Figure 1 shows $\log _{10}$ ratios of right to left responses in each of the six line orientations plotted as a function of the $\log _{10}$ ratios of reinforcers obtained on the right and left. The straight line fitted to the $\log$ ratios for $0^{\circ}$ by the method of least squares is described by Equation 1, and the lines fitted to log ratios for the other orientations are described by Equation 2. The slopes of the lines are given by $a_{11}$ (for $0^{\circ}$ ) and $a_{r 2}$ (other orientations). The intercepts are $\log c+\log d_{1}$ for $0^{\circ}$ and $\log c-\log d_{2}$ for $15^{\circ}$ through $75^{\circ}$. The variance in the data accounted for by the bestfitting straight lines ranged from .53 to .96 , and the standard errors of the estimate for orientations $0^{\circ}$ through $75^{\circ}$, respectively, were $.25, .11, .17, .08, .06$, and .14 for Bird G4 and .23, .24, .42, .28, .20, and .27 for Bird G5. Measures of discriminability of each orientation from $0^{\circ}$ were obtained following Davison and Tustin's (1978) analysis by subtracting Equation 2 from Equation 1 (assuming $a_{r 1}=a_{r 2}$ ) to give

$$
\log d=.5\left[\log d_{1}+\log d_{2}\right] .
$$

For $0^{\circ}, \log c+\log d_{1}$ was 2.17 for Bird G4 and 2.27 for Bird G5. Subtracting the intercept for each of the other orientations, $\log c-\log d_{2}$, from $\log c+\log d_{1}$ gave $\log d$ measures for the comparisons between $0^{\circ}$ and each other orientation. The $\log \mathrm{d}$ discriminability measures are shown in the lower panel of Figure 2 for each bird. Figure 2 clearly shows that as the orientation difference increased from $15^{\circ}$ to $75^{\circ}, \log \mathrm{d}$ increased. This result is generally consistent with the increase in discriminability with in- 


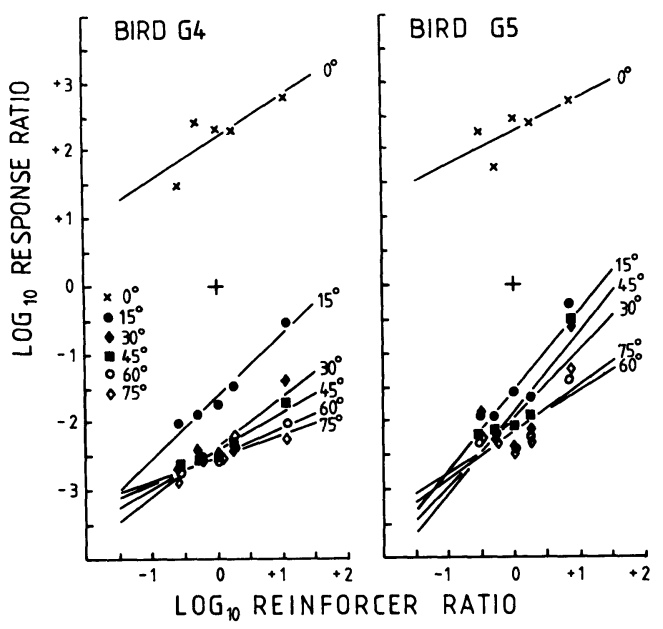

Figure 1. Logarithms of right-to-left response ratios in the presence of different line orientations, $0^{\circ}$ through $75^{\circ}$, as a function of logarithms of ratios of reinforcers obtained for right $\left(0^{\circ}\right)$ versus left (other orientations) responses. The large cross indicates the origin of each graph, and the straight lines are functions best fitting the $\log$ ratios for each orientation.
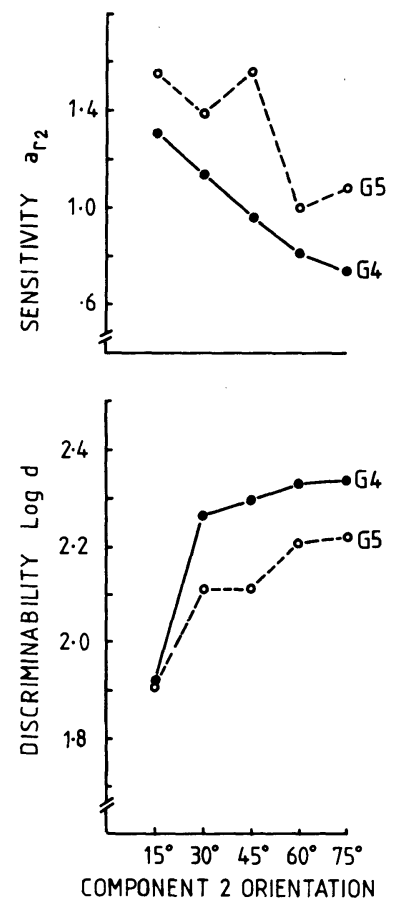

Figure 2. Discriminability of Component 2 orientations from $0^{\circ}$ (lower panel) and sensitivity to the right-to-left reinforcer ratio of right-to-left response ratios in the presence of each Component 2 orientation (upper panel). Discriminability values were derived from the intercepts of the best-fitting lines in Figure 1, and sensitivity values are the slopes of the lines for $15^{\circ}$ through $75^{\circ}$.

creasing difference between duration stimuli reported by McCarthy and Davison (1980b) and, in particular, is consistent with White et al.'s (1983) conclusion that an increase in the power-function exponent for multiple sched- ules may be related to an increase in the discriminability of the stimuli signaling the components.

Davison and Tustin's (1978) analysis assumes that the values for sensitivity to reinforcement in Equations 1 and 2 above, $\mathrm{a}_{\mathrm{r} 1}$ and $\mathrm{a}_{\mathrm{r} 2}$, are equal. Indeed, McCarthy and Davison have reported several failures to find consistent differences between $a_{r 1}$ and $a_{r 2}$ and have argued that the magnitude of $a_{\mathrm{r} 1}$ and $\mathrm{a}_{\mathrm{r} 2}$ should be unrelated to stimulus difference (McCarthy \& Davison, 1980a; McCarthy, Davison, \& Jenkins, 1982). For the present data, slopes of the functions for $0^{\circ}\left(\mathrm{a}_{11}\right)$ were .62 and .50 for Birds G4 and G5, respectively. Slopes of the functions in Figure 1 for each other orientation are shown in the upper panel of Figure 2 for each bird. Figure 2 shows that as the Component 2 orientation became increasingly different from $0^{\circ}$, values for $a_{12}$ decreased. Furthermore, the reduction in $a_{r 2}$ appeared to be a mirror image of the increase in $\log d$ with stimulus difference. Figure 2 suggests, therefore, that discriminability and sensitivity to reinforcement may not be independent. The problem of nonindependence most likely stems from the main assumption expressed in Equations 1 and 2, namely, that the choice between right and left in the presence of one orientation is determined by right and left reinforcers in all orientations. For example, any tendency to choose right in $15^{\circ}$ (although only left responses in $15^{\circ}$ are reinforced) is influenced by reinforcers for choosing right in the $0^{\circ}$ orientation. But, as the other orientations become more distant from $0^{\circ}$, changes in the reinforcers for right responses in the $0^{\circ}$ orientation may have progressively weaker effects on responding in other orientations. The model for measuring discriminability may therefore have to take into account the possibility that the effects of reinforcers in one component of a conditional discrimination on responding in another are modulated by the discriminability of the component stimuli.

\section{REFERENCES}

BaUm, W. M. (1974). On two types of deviation from the matching law: Bias and undermatching. Journal of the Experimental Analysis of Behavior, 22, 231-242.

Davison, M. C., \& Tustin, R. D. (1978). The relation between the generalized matching law and signal-detection theory. Journal of the Experimental Analysis of Behavior, 29, 331-336.

MCCARTHY, D., \& DAVISON, M. (1980a). Independence of sensitivity to relative reinforcement rate and discriminability in signal detection. Journal of the Experimental Analysis of Behavior, 34, 273-284.

MCCARThY, D., \& Davison, M. (1980b). On the discriminability of stimulus duration. Journal of the Experimental Analysis of Behavior, 33, 187-211.

McCarthy, D., Davison, M., \& Jenkins, P. E. (1982). Stimulus discriminability in free-operant and discrete-trial detection procedures. Journal of the Experimental Analysis of Behavior, 37, 199-215.

White, K. G., Pipe, M.-E., \& Mclean, A. P. (1983). Dimensional stimulus control of multiple schedule performance. Behaviour Analysis Letters, 3, 51-57.

White, K. G., PiPe, M.-E., \& McLean, A. P. (1984). Stimulus and reinforcer relativity in multiple schedules: Local and dimensional effects on sensitivity to reinforcement. Journal of the Experimental Analysis of Behavior, 41, 69-81.

(Manuscript received for publication December 6, 1984.) 